\title{
EDITORIAL \\ Total sacrectomy for malignant sacral tumors via a posterior-only approach
}

\author{
Mohamad Bydon, MD, Rafael De la Garza-Ramos, MD, and Ziya L. Gokaslan, MD \\ Department of Neurosurgery, Johns Hopkins University School of Medicine, Baltimore, Maryland
}

$\mathrm{P}$ RIMARY malignant sacral tumors, such as chordoma and sarcomas, are rare. While some tumors such as Ewing's sarcoma may be amenable to nonoperative treatment, the best long-term disease-free survival is achieved via en bloc resection, typically via sacrectomy. ${ }^{4}$ Nevertheless, sacrectomies are challenging procedures for several reasons. First, the proximity of the sacrum to large vessels such as the iliac veins and arteries carries the risk of vascular injury. Second, the proximity of the sacrum to the rectum carries the risk of rectal injury. Third, extensive tumors often require the sacrifice of nerve roots critical for bowel and bladder function. Fourth, sacrectomies require complex instrumented reconstructions to support the load of the upper body.

Fourney et al. devised a surgical classification system to aid in planning of these operations. ${ }^{2}$ This system was based on the level of nerve root sacrifice, and classified sacrectomies as low, middle, high, and total. Low sacral amputations involved resection at the level of the S-4 nerve roots; midsacral amputations at the level of S-3; high sacral amputations at the level of S-2; and total sacrectomies involved sacrifice of the S1-5 nerve roots bilaterally.

Historically, sacrectomies were first performed via combined anterior-posterior approaches. ${ }^{2}$ In the first stage, the iliac vessels are separated and their branches are dissected away from the sacrum and ligated. This is followed by soft-tissue dissection (including the rectum) and discectomy at the L5-S1 level. Afterward, the posterior approach involves removal of the sacrum by disruption of the sacroiliac joint and reconstruction via the use of lumbar transpedicular screws, iliac screws, rods, bars, and/or others. However, this procedure is associated with long operative times and significant trauma to the patient.

To overcome these issues, a posterior-only approach was developed for tumors that did not invade the pelvic organs nor extend beyond the lumbosacral junction. ${ }^{1-3}$ This technique utilizes a "midline posterior approach coupled with bilateral iliac osteotomies and midline osteotomy or discectomy and transperineal dissection, allowing deliv- ery of the en bloc sacral specimen."' In one of the largest series to date, Clarke et al. performed 36 primary posterior-only en bloc sacral resections, most of them for sacral chordomas. ${ }^{1}$ There were 13 complications (36.1\%), including $9(25.0 \%)$ wound infections/revisions. Furthermore, the nerve roots sacrificed ranged from preservation of the S-4 nerve roots and above, to bilateral sacrifice of S-1 and all nerve roots below. The performed operations included 2 total, 8 high, 9 middle, 12 low, and 5 distal sacrectomies.

In this issue of Journal of Neurosurgery: Spine, Zang et al. report outcomes of 10 patients undergoing total sacrectomy for en bloc resection of primary malignant sacral tumors via a posterior-only approach. ${ }^{5}$ This retrospective study took place at the Musculoskeletal Tumor Center of Peking University People's Hospital in China, and involved patients treated between July 2011 and July 2013. The tumors treated included 4 chordomas, 2 Ewing's sarcomas, 1 chondrosarcoma, 1 osteosarcoma, 1 malignant schwannoma, and 1 malignant giant cell tumor. The mean age of all patients was 35 years, and the most common presenting symptom was sacrococcygeal pain with radiation to the buttocks and lower limbs. Additionally, patients had bowel/bladder dysfunction.

The mean duration of surgery was 282 minutes, and the median estimated blood loss was $2595 \mathrm{ml}$. The complication rate was $40 \%$ (3 wound infections and 1 deep vein thrombosis). Patients were followed up for a mean of 22 months. Adequate margins were achieved in 8 patients; there were 2 recurrences (20\%), 1 in a patient with osteosarcoma with adequate margins (10 months after surgery) and 1 in a patient with Ewing's sarcoma with inadequate margins (6 months after surgery). There were 2 deaths due to disease progression: one case of osteosarcoma and another of Ewing's sarcoma. Among the surviving 8 patients, 7 patients emptied their bladder via catheterization and 1 by manually applying pressure to the abdomen. All patients experienced varying degrees of constipation, but none required a colostomy. At last follow-up, 6 of the 8 surviving patients were able to walk without any assistive 
devices, and 2 required external support. The 5-year overall survival rate by Kaplan-Meier analysis was $70 \%$.

The work presented herein has inherent merits, and the authors should be congratulated on such an effort. It is important to note that there are several issues that could have potentially biased the results. First, the heterogeneity of tumors included could be a potential source for bias. This might have confounded the 70\% 5-year overall survival, and results must be interpreted with caution. Second, the small sample size of 10 patients limits the ability to draw strong conclusions. Third, another possible confounder of survival is the fact that average follow-up time was only 22 months, and survival might have been much lower if patients had been followed for longer periods of time. The authors do not comment on how the surgical incision was closed, and in our experience the use of a myocutaneous rectus flap with the assistance of plastic surgery provides excellent results. Larger future studies focusing on total sacrectomy as a primary treatment modality for resection of a specific tumor histology will improve evidence quality.

http://thejns.org/doi/abs/10.3171/2014.11.SPINE141068

\section{References}

1. Clarke MJ, Dasenbrock H, Bydon A, Sciubba DM, McGirt MJ, Hsieh PC, et al: Posterior-only approach for en bloc sacrectomy: clinical outcomes in 36 consecutive patients. Neurosurgery 71:357-364, 2012

2. Fourney DR, Rhines LD, Hentschel SJ, Skibber JM, Wolinsky JP, Weber KL, et al: En bloc resection of primary sacral tumors: classification of surgical approaches and outcome. J Neurosurg Spine 3:111-122, 2005

3. Sahakitrungruang C, Chantra K, Dusitanond N, Atittharnsakul P, Rojanasakul A: Sacrectomy for primary sacral tumors. Dis Colon Rectum 52:913-918, 2009

4. Varga PP, Szövérfi Z, Lazary A: Surgical treatment of primary malignant tumors of the sacrum. Neurol Res 36:577-587, 2014

5. Zang J, Guo W, Yang R, Tang X, Li D: Is total en bloc sacrectomy using a posterior-only approach feasible and safe for patients with malignant sacral tumors? J Neurosurg Spine [epub ahead of print March 27, 2015. DOI: 10.3171/2015.1.SPINE14237]

\section{Response}

\section{Wei Guo, MD, PhD}

Musculoskeletal Tumor Center, People's Hospital, Peking University, Beijing, China

We would like to thank Dr. Bydon and colleagues for their insightful and thoughtful comments on our study. The goal of treating most primary sacral malignancies is aggressive en bloc resection with adequate surgical margins as most of the tumors are resistant to conventional radiotherapy and chemotherapy. The main purpose of our study was to introduce our preliminary experience with posterior-only total en bloc sacrectomy. We have performed this procedure at our institution since July 2011 after accumulating surgical experience from more than 500 sacrectomy cases performed via combined anteriorposterior approaches for 8 years.

Indications for such a procedure are relatively limited. Strict patient selection is imperative with special atten- tion to evaluation of iliac vessels and lumbosacral involvement, and also the relationship between tumor mass and visceral organs. Key structures (iliac vessels and the rectum) were bluntly dissected using moist gauze packed gradually and then pushed forward away from the sacrum. The dissected space between the anterior surface of tumor or sacrum and visceral organs was then filled with gauze. The space can be used to place the silicone tube, which can be later used as the guide for the Gigli saw for osteotomy and also can protect the lumbosacral trunk, internal iliac vessels, and rectum from the iliac osteotomy. The vessels and nerves can then be visualized after lifting the sacrum and being further manipulated.

The small number of patients and comparatively short follow-up time represent the main limitations of our study. We agree that several factors confounded the 5-year survival. In our paper, we provide an overview of the survival outcomes of this small group of patients with primary sacral malignancy after receiving the procedure. A larger number of patients with longer follow-up will provide a higher quality of oncological outcomes after total sacrectomy.

A myocutaneous flap was not routinely used after sacrectomy at our institution except in patients who had undergone preoperative radiotherapy. Dead space, proximity to the perineal region, poor blood supply, and neural atrophy may put patients with sacral tumors at high risk for wound complications. In 2003, we reported the largest series of wound complications after surgery for sacral tumors in 387 patients. The surgical site infection rate was $13.2 \%$, and wound dehiscence developed in $16 \%$ of patients. The results did not reveal a significantly higher wound complication rate than series with soft-tissue reconstruction reported in the literature. ${ }^{1}$ Additionally, adequate drainage is very important during postoperative care. Two drains were routinely used and were not removed until the drainage was less than $30 \mathrm{ml}$ in 24 hours to ensure adequate drainage. Some authors report placing an AlloDerm sling or bovine pericardial patch. In contrast, we did not place any materials on the anterior surface of the sacrum. The rectum was left to fill the defect resulting from the removal of sacral tumor. Thus, the dead space was reduced and the infection rate was decreased. Also we found that the wound complication rate was lower in patients who underwent total sacrectomy than in those undergoing transverse partial sacrectomy (unpublished data). The lower wound complication rate was due to better flap perfusion. We speculated that a larger bone defect in the sacrum causes less pressure on the wound flap in the supine position. In addition, an airbed is used to reduce the risk of pressure sores. Further investigation is warranted to prove our speculation.

Our hope is that exploring such a procedure will provide the groundwork necessary for improving clinical outcome of patients with primary sacral malignancies in order to meet the goal of performing a procedure with reduced trauma as well as adequate margins in these complicated and challenging cases.

\section{Reference}

1. Li D, Guo W, Qu H, Yang R, Tang X, Yan T, et al: Experience with wound complications after surgery for sacral tumors. Eur Spine J 22:2069-2076, 2013 